\title{
Persistent pulmonary hypertension and right ventricular function after percutaneous mechanical thrombectomy in severe acute pulmonary embolism
}

To the Editor:

Acute pulmonary embolism is one of the main causes of death, morbidity and hospitalisation [1, 2]. The most common cause of early death in these patients is right ventricular failure [1-3]. Complete resolution of the pulmonary thrombus at 6 months of evolution is not achieved in $57 \%$ of patients, with persistent right ventricular dysfunction (RVD) in $6-8 \%$ of patients and persistent pulmonary arterial hypertension (PAH) in 7-13.5\% of patients who received treatment with heparin or fibrinolytic agents [4-8]. Percutaneous mechanical thrombectomy (PMT) is accepted as a treatment in patients with high-risk acute pulmonary embolism with contraindications to systemic thrombolysis or when the treatment has failed $[1,2,9]$.

The main objective of this study was to analyse right ventricular function and the frequency of persistent PAH for up to 24 months or more in patients with acute pulmonary embolism and RVD treated with PMT.

We included patients (from July 2004 to June 2014) with diagnoses of severe acute pulmonary embolism with one or more criteria of RVD by echocardiography (hypokinesis of the free right ventricular wall, right ventricular diastolic diameter (RVDD)/left ventricular diastolic diameter ratio $>1$, RVDD $>30 \mathrm{~mm}$, tricuspid annular plane systolic excursion (TAPSE) $<17 \mathrm{~mm}$ or increased tricuspid regurgitation jet velocity); shock index $>1$; simplified pulmonary embolism severity index $>1$; positive cardiac biomarker; and evidence of thrombus in the central arteries and/or lobar branches from angiotomography or magnetic resonance imaging. Exclusion criteria were prior PAH, right ventricular hypertrophy and less than 6 months of follow-up. The PMT was indicated in patients with failed thrombolysis (persistent clinical instability and residual RVD on echocardiography), a high risk of increased bleeding or a contraindication to thrombolytics [9]. Patients undergoing thrombus fragmentation, mechanical thrombus aspiration with or without "in situ" thrombolysis or a combination of both methods were included. The patients were evaluated clinically and by echocardiography at 6 months and then once per year. Right ventricular function was evaluated by echocardiography. PAH was defined as levels of pulmonary systolic arterial pressure (PSAP) $\geqslant 40 \mathrm{mmHg}$ or $\geqslant 25 \mathrm{mmHg}$ of the mean pulmonary artery pressure. The PSAP was estimated by peak tricuspid regurgitation jet velocity using the simplified Bernoulli equation and combining this value with the right atrial pressure that was estimated from inferior vena cava diameter and respiratory changes [3].

Continuous variables are presented as mean $\pm \mathrm{SD}$, and categorical variables as frequencies and percentages. Comparisons were made using Fisher's exact test and t-tests. Survival analysis was performed using the Kaplan-Meier method. p-values $<0.05$ were considered statistically significant. Analyses were carried out using the statistical package IBM SPSS Statistics for Macintosh Version 20.0 (IBM Corp., Armonk, NY, USA).

Of the 412 patients evaluated for acute pulmonary embolism during the study period, 78 patients underwent PMT and 52 of these were included in the study. We excluded eight patients for prior PAH, six for right ventricular hypertrophy and five for follow-up of less than 6 months. Patients who died in the hospitalisation phase were not included in the analysis $(n=7)$. The mean \pm sD patient age was $51.8 \pm 15.9$ years. The most common contraindication to thrombolysis was recent major surgery $(38.5 \%)$, and the most common risk factor was deep vein thrombosis (50\%).

@ERSpublications

Percutaneous mechanical thrombectomy in acute PE restores right ventricle function in most patients at 40 months http://ow.ly/lhTh304sqeY

Cite this article as: Eid-Lidt G, Gaspar J, Sandoval J, et al. Persistent pulmonary hypertension and right ventricular function after percutaneous mechanical thrombectomy in severe acute pulmonary embolism. Eur Respir J 2017; 49: 1600910 [https://doi.org/10.1183/13993003.00910-2016]. 
TABLE 1 Echocardiography

Characteristics

Right ventricular function

Baseline 6-month control p-value Final control p-value

$\begin{array}{lcccccc}\text { Subjects n } & 52 & & & & & \\ \text { RVDD mm } & 42.6 \pm 5.1 & 42.6 \pm 5.1 & 37.4 \pm 5.7 & 0.0001 & 35.1 \pm 5.9 & 0.018 \\ \text { RVH } & 52(100 \%) & 100 \% & 20.9 \% & 0.001 & 16.6 \% & 0.067 \\ \text { TAPSE mm } & 13.4 \pm 2.8 & 13.3 \pm 2.7 & 20.7 \pm 2.4 & 0.0001 & 22.0 \pm 2.9 & 0.016 \\ \text { PSAP mmHg } & 60.8 \pm 17.7 & 60.8 \pm 17.7 & 42.8 \pm 14.8 & 0.0001 & 34.6 \pm 12.1 & 0.001 \\ \text { PSSM } & 52(100 \%) & & & & & \\ \text { LVEF } & 59.9 \pm 6.9 \% & & & & & \\ \text { RV/LVEDD >1 } & 52(100 \%) & & & & \end{array}$

RVDD: right ventricular diastolic diameter; RVH: right ventricular hypokinesis; TAPSE: tricuspid annular plane systolic excursion; PSAP: pulmonary systolic arterial pressure; PSSM: paradoxical septal systolic motion; LVEF: left ventricular ejection fraction; RV/LVEDD: right ventricular/left ventricular end diastolic diameter ratio.

PMT was performed 4.3 \pm 3.1 days (range 1-15 days) after symptom onset. Thrombus fragmentation was the most frequently used procedure (95.2\%), followed by aspiration $(53.8 \%)$ and the combination of both methods in $44.2 \%$ of patients. A permanent filter was implanted in the inferior vena cava in $46(88.5 \%)$ out of 52 patients.

After the procedure, the shock index (pre-procedure $1.1 \pm 0.23$ versus post-procedure $0.7 \pm 0.1 ; p=0.019$ ), heart rate (pre-procedure $113 \pm 14$ versus post-procedure $86 \pm 13$ beats per min; $p=0.005$ ) and systolic systemic arterial pressure (pre-procedure $100 \pm 14$ versus post-procedure $124 \pm 13 \mathrm{mmHg} ; \mathrm{p}=0.005$ ) improved. No recurrence of pulmonary embolism was documented during in-hospital outcomes.

The mean \pm sD follow-up was $40.2 \pm 16.7$ months (range 11-84 months). During the first 6 months, follow-up was complete in 52 patients (100\%), and 48 (92.3\%) patients completed follow-up for $\geqslant 24$ months. Four patients were re-admitted to hospital, two patients for recurrence of severe pulmonary embolism (4.1\%) and two for complicated pneumonia. Reduction of PSAP was progressive, with PSAP $\geqslant 50 \mathrm{mmHg}$ documented in $34.6 \%$ of patients at 6 months and in only $4.1 \%$ of patients after 24 months (two out of 48 patients). Ventilation-perfusion lung scans and tomography studies were performed on all eight (16.7\%) patients with PSAP levels $\geqslant 40 \mathrm{mmHg}$ during the follow-up period. In two patients, diagnoses of chronic pulmonary embolism were completed. All patients received oral anticoagulation with acenocoumarol during the first 6 months of follow-up.

Improvements of right ventricular function were greater during the first 6 months. TAPSE increased $55.6 \%$, the RVDD was reduced $12.2 \%$, and the PSAP was reduced by $29.6 \%$. However, right ventricular function continued to improve after 6 months (table 1). Complete recovery of hypokinesis of the free right ventricular wall in the first 6 months was documented in $79.1 \%$ of patients. Overall survival (extra-hospital phase) at 5 years was $96.2 \%, 82.1 \%$ were in New York Heart Association functional class 1 and $83.6 \%$ were free from recurrence of severe pulmonary thromboembolism.

The main results of our study are as follows: 1) in $16.7 \%$ of our patients, PSAP $\geqslant 40 \mathrm{mmHg}$ was documented at 24 months; 2) $4.1 \%$ of patients with long-term follow-up presented PAH from chronic pulmonary embolism; and 3) improvement of right ventricular function occurred mainly during the first 6 months of evolution.

Ribeiro et al. [8] reported that $43 \%$ of patients treated with heparin and/or thrombolysis had residual PAH after 12 months of follow-up. In patients with submassive pulmonary embolism, KLINE et al. [6] demonstrated systolic right ventricular pressure $\geqslant 40 \mathrm{mmHg}$ at 6 months in $7 \%$ of patients who received heparin only and in $11 \%$ of those that received heparin and alteplase.

In our patients, PSAP $>50 \mathrm{mmHg}$ at 42 months was low (4.1\%). The reduction in PSAP levels was higher during the first 6 months (29.6\%), with a further $21.4 \%$ reduction after this time. These results are consistent with the results of NIJKEUTER et al. [4], who observed complete resolution of thromboembolism by tomography in $43 \%$ of patients at 6 months.

The cumulative incidence rates of PAH from symptomatic chronic pulmonary embolism among patients with an acute episode of pulmonary embolism have been reported to be $3.8 \%$ at 2 years [10-12]. In our population with follow-up up of 24 months or more, the $\mathrm{PAH}$ incidence from chronic pulmonary thromboembolism was $4.1 \%$. 
Several studies have evaluated right ventricular function in patients with acute pulmonary embolism treated with heparin or fibrinolytic therapy. Meneveau et al. [5] showed that reversal of RVD occurs in $80 \%$ of patients within the first $48 \mathrm{~h}$ in patients with submassive and massive pulmonary embolism. Most of the contractile right ventricular deficit improves at 6 months [5, 13]. Ribeiro et al. [8] reported that $8.3 \%$ of patients persisted with RVD at 12 months of echocardiographic follow-up.

Long-term evaluations of right ventricular function in patients undergoing PMT have not been published. In the PERFECT (pulmonary embolism response to fragmentation, embolectomy, and catheter thrombolysis) trial, a significant improvement in right ventricular function in the acute phase was documented [14]. In our study, TAPSE increased $>50 \%$, and mobility of the free right ventricular wall was completely restored in most patients during the first 6 months. The recovery of the right ventricle persisted after 6 months, with a 9.1\% increase in TAPSE values and a $21.4 \%$ reduction in PSAP levels. The benefit of the PMT and local thrombolysis in patients with markedly elevated PSAP levels may be limited, suggesting chronic character of the pulmonary embolism. We excluded patients with prior PAH, right ventricular hypertrophy and/or clinical conditions related to PAH (i.e. obesity) to reduce this possibility.

The current study provides valuable information on the right ventricular function and incidence of PAH in patients with severe acute pulmonary embolism undergoing PMT with follow-up for longer than 24 months.

Guering Eid-Lidt ${ }^{1}$, Jorge Gaspar ${ }^{1}$, Julio Sandoval ${ }^{2}$, Hector Gonzalez-Pacheco ${ }^{3}$, Antonio Arias ${ }^{4}$, Pablo Acevedo ${ }^{1}$, Tomas Pulido ${ }^{2}$, Carlos Martinez-Sanchez ${ }^{3}$ and Marco Antonio Martinez-Rios ${ }^{1}$

${ }^{1}$ Interventional Cardiology, Instituto Nacional de Cardiologia Ignacio Chavez, Mexico City, Mexico. ${ }^{2}$ Cardiopulmonary Dept, Instituto Nacional de Cardiologia Ignacio Chavez, Mexico City, Mexico. ${ }^{3}$ Coronary Care Unit, Instituto Nacional de Cardiologia Ignacio Chavez, Mexico City, Mexico. ${ }^{4}$ Echocardiography, Instituto Nacional de Cardiologia Ignacio Chavez, Mexico City, Mexico.

Correspondence: Guering Eid-Lidt, Interventional Cardiology, Instituto Nacional de Cardiologia Ignacio Chavez, Juan Badiano 1, Mexico City, 14080, Mexico. E-mail: gueringeidlidt@gmail.com

Received: March 222016 | Accepted after revision: Sept 112016

Conflict of interest: None declared.

\section{References}

1 Konstantinides SV, Torbicki A, Agnelli G, et al. 2014 ESC Guidelines on the diagnosis and management of acute pulmonary embolism. Eur Heart J 2014; 35: 3033-3073.

2 Jaff M, McMurtry S, Archer SL, et al. Management of massive and submassive pulmonary embolism, iliofemoral deep vein thrombosis, and chronic thromboembolic pulmonary hypertension: a scientific statement from the American Heart Association. Circulation 2011; 123: 1788-1830.

3 Galiè N, Humbert M, Vachiery J-L, et al. 2015 ESC/ERS Guidelines for the diagnosis and treatment of pulmonary hypertension. Eur Heart J 2016; 37: 67-119.

4 Nijkeuter M, Hovens MMC, Davidson BL, et al. Resolution of thromboemboli in patients with acute pulmonary embolism: a systematic review. Chest 2006; 129: 192-197.

5 Meneveau N, Ming LP, Seronde MF, et al. In-hospital and long-term outcome after sub-massive and massive pulmonary embolism submitted to thrombolytic therapy. Eur Heart J 2003; 24: 1447-1454.

6 Kline JA, Steurwald MT, Marchick MR, et al. Prospective evaluation of right ventricular function and functional status 6 months after acute submassive pulmonary embolism: frequency of persistent or subsequent elevation in estimated pulmonary artery pressure. Chest 2009; 136: 1202-1210.

7 de Perrot M, Fadel E, McRae K, et al. Evaluation of persistent pulmonary hypertension after acute pulmonary embolism. Chest 2007; 132: 780-785.

8 Ribeiro A, Lindmarker P, Johnsson $\mathrm{H}$, et al. Pulmonary embolism: one-year follow-up with echocardiography doppler and five-year survival analysis. Circulation 1999; 99: 1325-1330.

9 Eid-Lidt G, Gaspar J, Sandoval J, et al. Combined clot fragmentation and aspiration in patients with acute pulmonary embolism. Chest 2008; 134: 54-60.

10 Moores L, Zamarro C, Gómez V, et al. Changes in PESI scores predict mortality in intermediate-risk patients with acute pulmonary embolism. Eur Respir J 2013; 41: 354-359.

11 Pengo V, Lensing AW, Prins MH, et al. Incidence of chronic thromboembolic pulmonary hypertension after pulmonary embolism. N Engl J Med 2004; 350: 2257-2264.

12 Becattini C, Agnelli G, Pesavento R, et al. Incidence of chronic thromboembolic pulmonary hypertension after a first episode of pulmonary embolism. Chest 2006; 130: 172-175.

13 Golpe R, Testa-Fernández A, Pérez-de-Llano LA, et al. Long-term clinical outcome of patients with persistent right ventricle dysfunction or pulmonary hypertension after acute pulmonary embolism. Eur J Echocardiogr 2011; 12: 756-761.

14 Kuo WT, Banerjee A, Kim PS, et al. Pulmonary embolism response to fragmentation, embolectomy, and catheter thrombolysis (PERFECT): initial results from a prospective multicenter registry. Chest 2015; 148: 667-673. 